\title{
Fullerene and fullerene-aluminum nanostructured films as sensitive layers for gas sensors
}

\author{
D. Grynko, J. Burlachenko, O. Kukla, I. Kruglenko, O. Belyaev \\ V. Lashkaryov Institute of Semiconductor Physics, NAS of Ukraine, \\ 41, prospect Nauky, 03028 Kyiv, Ukraine \\ Phone: (380-44) 525-56-26; fax: (380-44) 525-83-42; e-mail: kruglenko@yahoo.com
}

\begin{abstract}
The responses of quartz crystal microbalance (QCM) sensors coated by fullerene and fullerene-aluminum films to ethanol and water vapors are investigated. The possibility of controlling the adsorption properties of such films by inserting the aluminum and by ultraviolet irradiation that changes the film morphology in a definite way is demonstrated.
\end{abstract}

Keywords: Electronic Nose (QCM), fullerene nanostructured films, ultraviolet irradiation.

Manuscript received 08.04.09; accepted for publication 14.05.09; published online 30.06.09.

\section{Introduction}

A wide variety of nanostructured materials are among the most important objects of investigation in modern science. In particular, they are of significant interest for gas sensing applications. Any sensor system is characterized by certain functionality and selectivity profile, which defines its application area. Since the adsorption properties of sensors depend on their surface morphology and chemical composition, the investigation of new materials with well-defined surface structure is of great importance.

In this work, we discuss the advantages of using the fullerenes and fullerene-aluminum films as sensitive coatings for gas sensors.

Fullerenes and fullerene-based compounds are promising materials for various applications in analytical chemistry. They are used as chromatographic stationary phases, as electrochemical sensors based on their activity as electron mediators, as sorbent materials in continuous flow systems [1]. Great attention is paid to the use of fullerene and fullerene-based films as sensitive layers for chemical sensors. A lot of results concerned with the investigation of adsorption properties of such films towards various organic and inorganic compounds were reported in recent years. High sensitivity of masssensitive sensors coated by $\mathrm{C}_{60}$ film to humidity was shown in [2], while in the work [3] the humidity was considered as a negative factor that decreases the electrical response of the $\mathrm{C}_{60}$ film on hydrogen. The possibility to use $\mathrm{C}_{60}$ and their derivatives to detect various organic molecules like amines, dithiols, alkynes and 1,3-dienes (chemisorption) and alcohols, aldehydes, carboxylic acids (physical adsorption) as well as metal ions and anions with good reproducibility and detection limit was demonstrated in the work [4]. $\mathrm{C}_{60}$-based coatings of mass-sensitive biosensors are useful for the detection of hemohlobine, immunoglobulin G [5] and for detection of anti-hemoglobin and anti-myoglobin antibodies [6]. The perspectives of using the twocomponent films composed of fullerenes and some transitive metals for electrochemical sensors were discussed in the work [7].

It is known that morphology of fullerene and fullerene-based films can be changed in the definite way by creation of mixed metal-fullerene layers [7,8]. Another promising way of surface modification is application of some external influence, for example, illumination. The morphology and composition of photopolimeryzed $\mathrm{C}_{60}$ films was reported in [9].

In this work, the adsorption properties of fullerene $\mathrm{C}_{60}$ and fullerene-aluminum films towards ethanol and water vapours are investigated and discussed.

\section{Experimental procedure}

Mass-sensitive sensors based on quartz crystal microbalance (QCM) were used for the investigation of adsorption properties of fullerene and fullerenealuminum films. Sensors were put into the flow-type 
chamber. Saturated vapour of analyte passed through the chamber with a constant speed, argon was used as a carrier gas. The QCM oscillating frequency shift due to adsorption of analyte molecules on their surface was measured [10].

Fullerene and fullerene-aluminum films were deposited by thermal evaporation in vacuum directly on the electrode of QCM. Since the sublimation temperature of fullerenes is less than the aluminum evaporation temperature, there were used two different evaporators.

Morphology of films was studied using atomic force microscopy.

\section{Results and discussion}

The difference between fullerene and fullerenealuminum films can be seen from the AFM images shown in Fig. 1. The introduction of aluminum decreases the typical cluster size from 70-100 $\mathrm{nm}$ for the fullerene film down to 20-30 nm for the fullerene-aluminum one. At the same time, the uniformity of the surface is improved. The adsorption properties of two films significantly differ.

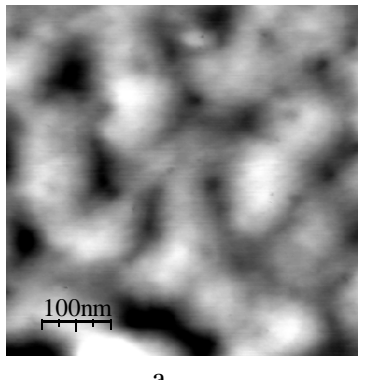

a

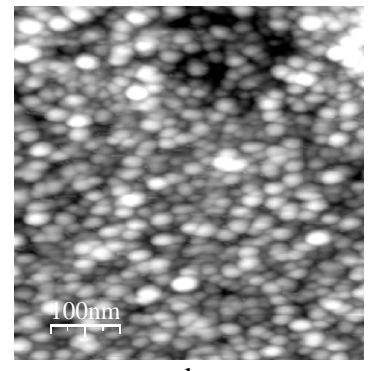

b
Fig. 1. AFM images of fullerene (a) and fullerene-aluminum (b) films.

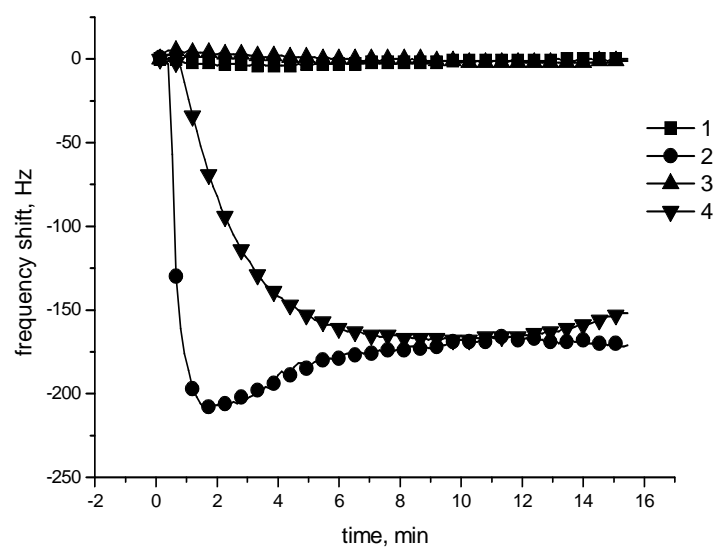

Fig. 2. The response of QCM sensors coated by fullerene ( 1 - to ethanol, 3 - to water vapour) and fullerene-aluminum ( 2 - to ethanol, 4 - to water vapour).
The responses of $\mathrm{C}_{60}$ film to water and ethanol average $3-5 \mathrm{~Hz}$ that is quite low for the adequate measurements (see Fig. 2, curves 1,3). The sensitivity of $\mathrm{C}_{60}-\mathrm{Al}$ film is about 55 times higher and totals $208 \mathrm{~Hz}$ for ethanol and $167 \mathrm{~Hz}$ for water vapour (curves 2 and 4 in Fig. 2). The increase of sensor response can be explained in view of the film structure: adsorption capacity of the nanoorganized metal-fullerene film is higher than that of fullerene film composed of relatively large clusters.

The similar influence on morphology and adsorption properties of $\mathrm{C}_{60}$ and $\mathrm{C}_{60}$ - $\mathrm{Al}$ films has their exposure to UV irradiation. With the increase of exposure time, the characteristic cluster size decreases, the surface becomes more uniform, and its sensitivity to water and ethanol vapours increases.

The dependence of the response for the $\mathrm{C}_{60}$-covered sensor to ethanol on the time of exposure to UV irradiation is shown in Fig. 3.

AFM images of fullerene (a) and fullerenealuminum (b) films after UV irradiation for $30 \mathrm{~min}$ are presented in Fig. 4. After $30 \mathrm{~min}$ UV irradiation, the amplitudes of the responses are about 50 to 90 times increased for $\mathrm{C}_{60}$ coating and about 2.5 times for the $\mathrm{C}_{60^{-}}$ $\mathrm{Al}$ one (see Fig. 5).

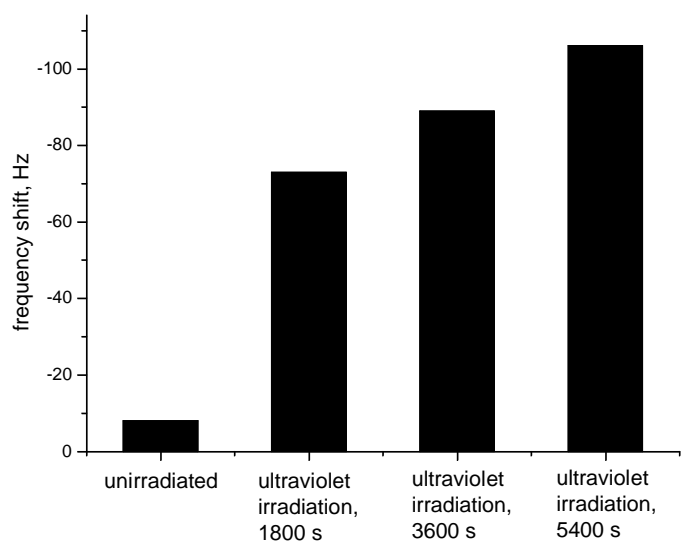

Fig. 3. The dependence of $\mathrm{C}_{60}$-coated QCM sensor response to ethanol vapor on the time of exposure to UV irradiation.

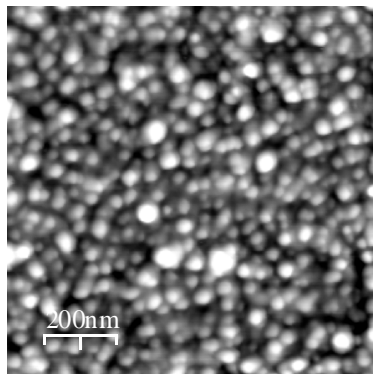

a

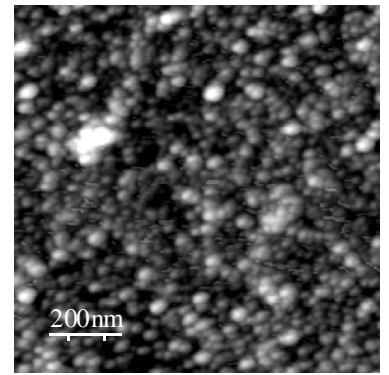

b
Fig. 4. AFM images of fullerene (a) and fullerenealuminum (b) films after UV irradiation for $30 \mathrm{~min}$. 


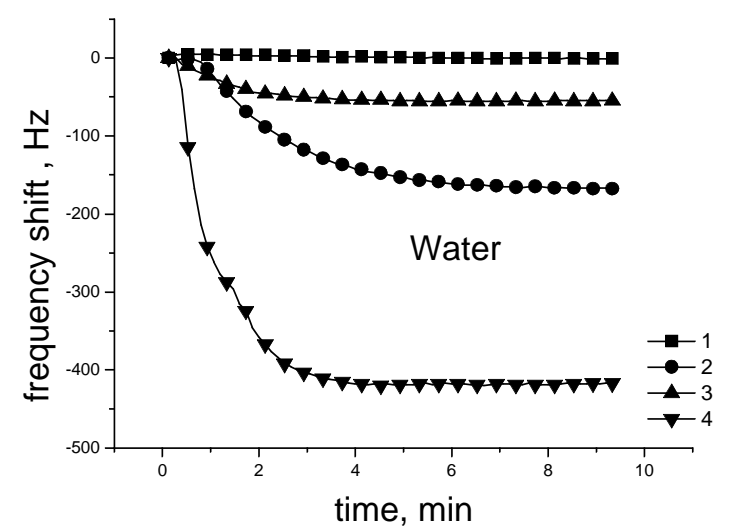

a

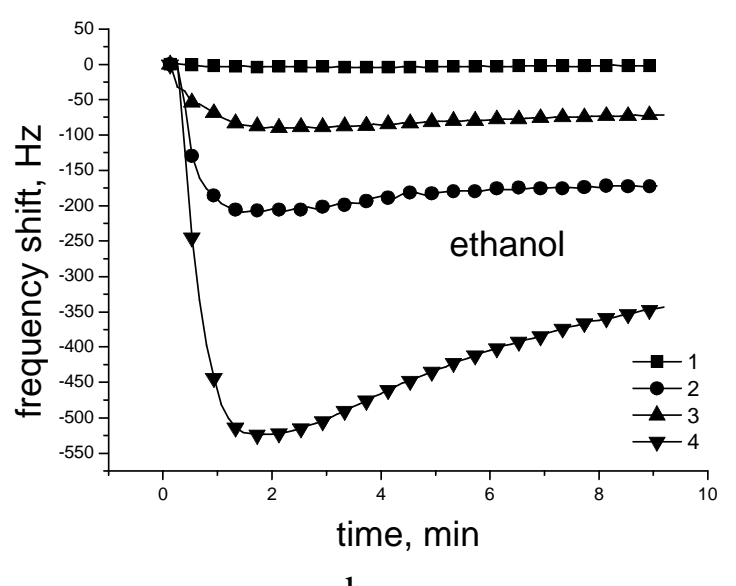

b

Fig. 5. QCM sensors' responses to water (a) and to ethanol (b) and for fullerene-coated sensors ( 1 - before UV irradiation, 3 after UV irradiation) and for fullerene-aluminum coated sensors ( 2 - before UV irradiation, 4 - after UV irradiation).

\section{Conclusions}

Modification of fullerene films by inserting metal atoms and/or UV irradiation is a promising way to increase the sensitivity of films to some analytes. Besides, the selectivity profile of the $\mathrm{C}_{60}$ film can be changed by illumination: the influence of UV exposure on the response amplitudes differs for water and ethanol. Both methods of $\mathrm{C}_{60}$ surface modification can be successfully used for the development of sensor arrays for gas analysis.

\section{Acknowledgments}

This work was supported by Program of the National Academy of Sciences of Ukraine "Nanosystems, Nanomaterials and Nanotechnologies".

\section{References}

1. J.R. Baena, M. Gallego, M. Valca'rcel, Fullerenes in the analytical sciences // Trends in analytical chemistry 21, No. 3, p. 187-198 (2002).

2. E. Radeva, V. Georgiev, L. Spassov, N. Koprinarov, St. Kanev, Humidity adsorptive properties of thin fullerene layers studied by means of quartz micro-balance // Sens. Actuators B: Chem. 42, p. 11-13 (1997).

3. G. Sberveglieri, G. Faglia, C. Perego, P. Nelli, R.N. Marks, T. Virgili, C. Taliani, R. Zamboni, Hydrogen and humidity sensing properties of $\mathrm{C}_{60}$ thin films // Synthetic Metals, 77, p. 273-275(1996).

4. Jeng-Shong Shih, Yun-Ching Chao, Mine-Fag Sung, Guann-Jou Gau and Chyow-San Chiou, Piezoelectric crystal membrane chemical sensors based on fullerene $\mathrm{C}_{60} / /$ Sens. Actuators $B$ : Chem.76, No. 1-3, p .347-353 (2001).

5. Nai-Yu Pan, Jeng-Shong Shih, Piezoelectric crystal immunosensors based on immobilized fullerene $\mathrm{C}_{60}$-antibodies // Sens. Actuators B: Chem. 98, p. 180-187(2004).

6. Hung-Wei Chang, Jeng-Shong Shih, Surface acoustic wave immunosensors based on immobilized $\mathrm{C}_{60}$-proteins // Sens. Actuators B: Chem. 121, p. 522-529 (2007).

7. Krzysztof Winkler, Alan L. Balch, Electrochemically formed two-component films comprised of fullerene and transition-metal components // C. R. Chimie 9, p. 928-943 (2006).

8. F.A. Khalid, O. Beffort, U.E. Klotz, B.A. Keller, P. Gasser, S. Vaucher, Study of microstructure and interfaces in an aluminum- $\mathrm{C}_{60}$ composite material // Acta Materialia 51, p. 4575-4582 (2003).

9. E. Alvarez-Zauco, H. Sobral, E.V. Basiuk, J.M. Saniger-Blesa, M. Villagra'n-Muniz, Polymerization of $\mathrm{C}_{60}$ fullerene thin films by $\mathrm{UV}$ pulsed laser irradiation // Appl. Surf. Sci. 248, p. 243-247 (2005).

10. I. Kruglenko, B. Snopok, Nonexponential relaxations in sensor arrays: forecasting strategy for electronic nose performance // Sens. Actuators B: Chem. 106, No. 1, p. 101-113 (2005). 